\title{
The effect of test meal monounsaturated fatty acid : saturated fatty acid ratio on postprandial lipid metabolism
}

\author{
Helen M. Roche ${ }^{1 *}$, Antonis Zampelas ${ }^{2}$, Kim G. Jackson ${ }^{3}$, Christine M. Williams ${ }^{3}$ \\ and Michael J. Gibney ${ }^{1}$ \\ ${ }^{1}$ Unit of Nutrition, Trinity Centre for Health Sciences, St James's Hospital, James's Street, Dublin 8, Ireland \\ ${ }^{2}$ School of Biological Sciences, University of Surrey, Guildford GU2 5XH, UK \\ ${ }^{3}$ Hugh Sinclair Unit of Human Nutrition, Department of Food Science and Technology, University of Reading, \\ Reading RG6 6AP, UK
}

(Received 30 May 1997-Revised 27 November 1997-Accepted 3 December 1997)

\begin{abstract}
Epidemiological evidence shows that a diet high in monounsaturated fatty acids (MUFA) but low in saturated fatty acids (SFA) is associated with reduced risk of CHD. The hypocholesterolaemic effect of MUFA is known but there has been little research on the effect of test meal MUFA and SFA composition on postprandial lipid metabolism. The present study investigated the effect of meals containing different proportions of MUFA and SFA on postprandial triacylglycerol and non-esterified fatty acid (NEFA) metabolism. Thirty healthy male volunteers consumed three meals containing equal amounts of fat $(40 \mathrm{~g})$, but different proportions of MUFA (12, 17 and $24 \%$ energy) in random order. Postprandial plasma triacylglycerol, apolipoprotein B-48, cholesterol, HDL-cholesterol, glucose and insulin concentrations and lipoprotein lipase (EC 3.1.1.34) activity were not significantly different following the three meals which varied in their levels of SFA and MUFA. There was a significant difference in the postprandial NEFA response between meals. The incremental area under the curve of postprandial plasma NEFA concentrations was significantly $(P=0.03)$ lower following the high-MUFA meal. Regression analysis showed that the non-significant difference in fasting NEFA concentrations was the most important factor determining difference between meals, and that the test meal MUFA content had only a minor effect. In conclusion, varying the levels of MUFA and SFA in test meals has little or no effect on postprandial lipid metabolism.
\end{abstract}

Fat intake: Monounsaturated fatty acids: Saturated fatty acids: Postprandial metabolism

An elevated postprandial lipaemic response is increasingly being recognized as an important risk factor for the development of CHD. Patients with CHD show a more prolonged postprandial triacylglycerolaemic response compared with healthy controls (Patsch et al. 1993) and the concentration of postprandial chylomicron remnant apolipoprotein (apo) B-48 has been directly related to the progression of atherosclerosis (Karpe et al. 1994). Recent prospective evidence has shown that non-fasting triacylglycerol concentrations are a significant predictor of future myocardial infarction (Stampfer et al. 1996) which further supports the hypothesis that impaired postprandial plasma triacylglycerol metabolism plays a causal role in the development and progression of CHD. The causal nature of this relationship is based on the central role of triacylgly- cerol-rich lipoprotein (TRL) metabolism, which influences the composition and metabolic fate of other lipid and lipoprotein fractions. TRL are atherogenic lipoproteins in their own right. Moreover, elevated postprandial TRL concentrations promote the catabolism of the cardio-protective HDL fraction, promote the formation of the highly atherogenic small dense LDL fraction (Roche \& Gibney, 1995) and influence plasma non-esterified fatty acid (NEFA) metabolism.

Although much of the interest in postprandial lipid metabolism has focused on the adverse consequences of elevated TRL, elevated plasma NEFA concentrations have been proposed as a risk marker of CHD (Frayn et al. 1996). Plasma NEFA and triacylglycerol metabolism are closely linked, both in the fasting and postprandial states. In the

\footnotetext{
Abbreviations: apo, apolipoprotein; AUC, area under the curve; IAUC, incremental area under the curve; MUFA, monounsaturated fatty acids; NEFA, nonesterified fatty acids; SFA, saturated fatty acids; TPL, triacylglycerol-poor lipoprotein; TRL, triacylglycerol-rich lipoprotein.

*Corresponding author: Dr Helen M. Roche, fax + 3531454 2043, email hmroche@tcd.ie
} 
fasting state, NEFA are released into the circulation from the hydrolysis of adipose tissue triacylglycerol; this is controlled by hormone-sensitive lipase ( $E C$ 3.1.1.3) activity (Coppack et al. 1994). Immediately following meal ingestion, plasma NEFA concentrations decline because insulin inhibits hormone-sensitive lipase activity, thereby preventing the release of NEFA from adipose tissue (Frayn et al. 1994). Following the initial fall in postprandial NEFA concentrations, plasma concentrations return to pre-meal concentrations. Much of this increase is due to reduced suppression of hormone-sensitive lipase with decreasing insulin concentration. In addition, a significant proportion (perhaps up to $50 \%$ ) of the hydrolysed postprandial TRL fatty acids are not taken up into adipose tissue but "spill over' into the circulation as NEFA, thus increasing plasma NEFA concentrations (Binnert et al. 1996). Plasma NEFA concentrations are the primary substrate for hepatic verylow-density lipoprotein (VLDL)-triacylglycerol synthesis; elevated NEFA concentrations induce hepatic VLDL synthesis thereby contributing to raised plasma triacylglycerol concentrations (Byrne et al. 1991). In view of the close metabolic relationship between NEFA and triacylglycerols, and their effect on the development and progression of CHD, dietary factors which modulate postprandial lipid metabolism need investigation. Virtually nothing is known of the effect of meal fat composition on postprandial NEFA metabolism. A large number of studies have evaluated the effect of fat composition on postprandial triacylglycerol metabolism (Weintraub et al. 1988; De Bruin et al. 1993; Zampelas et al. 1994), but have not measured NEFA.

Epidemiological evidence shows a low prevalence of CHD in Mediterranean regions (Keys et al. 1986) where the consumption of monounsaturated fatty acids (MUFA) is relatively high (Kafatos \& Mamalakis, 1993). The apparent cardio-protective effect of MUFA can only be partly explained by the hypocholesterolaemic effect of MUFA when substituted for saturated fatty acids (SFA) (Mensink \& Katan, 1989; Mensink, 1992); another possibility lies in their ability to modulate the postprandial lipaemic response. Therefore, the present study was designed to determine whether increasing meal MUFA content, at the expense of SFA, affects postprandial lipid metabolism, in an effort to explain the lower rates of CHD observed in populations consuming high amounts of MUFA.

\section{Methods}

Subjects

This study was approved by the Ethics Committee of the Federated Dublin Voluntary Hospitals in Ireland and the
Royal Surrey County Hospital in the UK, and the trial was conducted on an out-patient basis. Subjects gave written consent before participation in the trial. Thirty healthy male volunteers aged 22.8 (SD 2.9) years, weighing 74.0 (SD $8.3) \mathrm{kg}$ and with a mean BMI of $22.9(\mathrm{SD} 1.5) \mathrm{kg} / \mathrm{m}^{2}$ participated in the trial. The subjects were of stable body weight, habitually consumed between 34 and $45 \%$ energy as fat, were not adhering to a special diet and were not regular consumers of $n-3$ polyunsaturated fatty acid supplements or any other nutritional supplement. All participants were non-smokers, did not exercise excessively ( $>90 \mathrm{~min} /$ week) and were not being prescribed any medications.

\section{Test meal composition}

The acute test meals provided varying amounts of MUFA and SFA which reflected the fatty acid compositions of the typical northern European and traditional Cretan diets (Gregory et al. 1990; Kafatos \& Mamalakis, 1993). The medium-MUFA meal provided MUFA and SFA at levels typical of the current Athenian dietary fat composition. The test meal consisted of $135 \mathrm{~g}$ white bread, $36 \mathrm{~g}$ strawberry jam and a milk-shake consisting of $40 \mathrm{~g}$ of the test oil, $40 \mathrm{~g}$ dried skimmed milk powder (Tesco, Cheshunt, UK) and $40 \mathrm{~g}$ Nesquik strawberry flavoured milk-shake mix (Nestlé, Vevey, Switzerland), mixed with $200 \mathrm{ml}$ water (Évian, France). The fatty acid composition of the test meal is presented in Table 1. Subjects were randomly allocated to receive the high-, medium- and low-MUFA test meals once monthly for three consecutive months.

\section{Postprandial investigations}

Postprandial investigations of fifteen volunteers were completed at two centres, Trinity College Dublin and the University of Surrey. Plasma samples were re-distributed for central analysis. Each postprandial study began at between 07.30 and 08.00 hours following a $12 \mathrm{~h}$ overnight fast. All subjects abstained from alcohol consumption and refrained from strenuous exercise for $24 \mathrm{~h}$ before the postprandial investigation. During postprandial investigations subjects abstained from food (including chewing gum) and drinks, with the exception of caffeine-free, lowenergy $(<4.2 \mathrm{~kJ} / \mathrm{ml})$ drinks, e.g. decaffeinated black coffee, sugar-free drinks and water.

A 21 gauge, $32 \mathrm{~mm}$ venous catheter (Abbott Ireland Ltd, Dublin, Ireland) was inserted into the subject's antecubital vein of the non-dominant forearm. To ensure that the cannula remained patent throughout the $9 \mathrm{~h}$ study period it was flushed with sodium citrate in saline solution (Phoenix

Table 1. Fatty acid composition of the test meals (including the fat in the bread)

\begin{tabular}{lcccccc}
\hline & \multicolumn{3}{c}{ Fatty acids $(\mathrm{g} / 40 \mathrm{~g})$} & \multicolumn{2}{c}{ Fatty acids (\% energy) } \\
\cline { 2 - 4 } Test meal & SFA & MUFA & PUFA & SFA & MUFA \\
\hline Low-MUFA & 20.0 & 14.0 & 5.7 & 17.2 & 12.0 & PUFA \\
Medium-MUFA & 14.6 & 20.0 & 5.4 & 12.3 & 5.0 \\
High-MUFA & 6.3 & 28.3 & 5.4 & 5.4 & 4.6 \\
\hline
\end{tabular}

SFA, saturated fatty acids; MUFA, monounsaturated fatty acids, PUFA, polyunsaturated fatty acids. 
Pharmaceuticals Ltd, Gloucester, Glos., UK). Two fasting blood samples ( -10 and $0 \mathrm{~min}$ ) were drawn before the test meal was consumed. The test meal was consumed under supervision to ensure that all food was eaten within a $20 \mathrm{~min}$ period. Postprandial blood samples were drawn every hour for $9 \mathrm{~h}$. Blood samples were centrifuged $(2000 \mathrm{~g})$ immediately, the plasma was harvested and divided into two portions. One portion of plasma was vortex-mixed, divided into portions and frozen $\left(-20^{\circ}\right)$ immediately for subsequent analysis. The remaining plasma was used to prepare TRL and triacylglycerol-poor lipoprotein (TPL) fractions using the ultracentrifugation method, as previously described (Grundy \& Mok, 1976; Zampelas et al. 1994). This plasma was stored $\left(<5^{\circ}\right)$ for $24 \mathrm{~h}$, and ultracentrifugation was completed on the day after blood collection. Triacylglycerol, cholesterol and apoB-48 analysis was completed on each of these fractions as well as plasma.

After $9 \mathrm{~h}$ postprandial post-heparin lipoprotein lipase $(E C$ 3.1.1.34) activity and hepatic lipase activity ( $E C$ 3.1.1.3) were determined following the infusion of $7500 \mathrm{IU}$ heparin sodium (Multiparin Heparin Injection (5000 IU/ml), CP Pharmaceuticals Ltd, Wrexham, Clwyd, UK). Blood samples were drawn $5 \mathrm{~min}$ and $15 \mathrm{~min}$ following heparin injection. The blood samples were centrifuged $(2000 \mathrm{~g})$ immediately and the plasma was harvested, divided into portions and frozen $\left(-70^{\circ}\right)$ immediately for subsequent analysis. Fasting post-heparin lipoprotein lipase and hepatic lipase activities were also determined on a separate occasion, following an overnight fast $(12 \mathrm{~h})$.

\section{Biochemical variables investigated}

All analysis was completed within 12 months of the blood samples being drawn. Analysis of plasma, TRL and TPLtriacylglycerol (TAG PAP Uni-kit, Roche Diagnostics Ltd, Vevey, Switzerland), cholesterol (Cholesterol PAP Uni-kit, Roche Diagnotics Ltd) and NEFA (NEFA c Kit (ACS ACOD method), Wako Chemicals GmbH, Neuss, Germany) concentrations were determined using enzymic colorimetric assays on a Cobas-Bio centrifugal analyser (Cobas Mira, Roche Diagnostics Ltd). The inter-assay CV, measured with with external control plasma were $5.05 \%$ for plasma triacylglycerol, 3.66\% for TRL-triacylglycerol, $4.85 \%$ for plasma cholesterol and $8.58 \%$ for plasma NEFA.

ApoB-48 concentrations in plasma and the TRL fraction were determined using a competitive ELISA described previously (Lovegrove et al. 1996), using a specific polyclonal anti-apoB-48 antiserum (Peel et al. 1993). The inter-assay CV was $8.8 \%$ for $3.987 \mu \mathrm{g} / \mathrm{ml}$.

Lipoprotein lipase activity was determined using triolein as a substrate, by the method previously described (Nilsson-Ehle \& Schotz, 1976). Briefly, a triolein emulsion was diluted with $50 \mathrm{mM}$-Tris (containing $3 \mathrm{~g}$ bovine serum albumin/l and either $0.2 \mathrm{M}-\mathrm{NaCl}$ or $4 \mathrm{M}-\mathrm{NaCl}$ and $2 \mathrm{U}$ heparin/ml) and pre-incubation with inactivated human serum. Incubations of $20 \mu \mathrm{l}$ plasma with the emulsion were carried out for $30 \mathrm{~min}$ at $37^{\circ}$. A milk lipoprotein lipase standard was used to evaluate the assay. The interassay $\mathrm{CV}$, using a single emulsion, was $5.06 \%$ for $12800 \mathrm{mU} / \mathrm{ml}$.

\section{Statistical analysis}

All statistical analyses were completed with the Apple Macintosh-compatible statistical package Data Desk 4.1 (Data Description Inc., New York, NY, USA). Two fasting samples were analysed for all biochemical variables and the mean of these two values was used for statistical analysis. Plasma triacylglycerol, TRL-triacylglycerol and plasma apoB-48 concentrations were transformed to the natural log (ln) to give the data a normal Gaussian distribution. Repeated-measures ANOVA, with meal MUFA level as the independent variable, was employed to investigate differences in the postprandial response between meals.

The postprandial data were expressed in summary form, i.e. area under the postprandial response curve (AUC), the incremental area under the postprandial response curve (IAUC), maximum postprandial levels and time to maximal postprandial levels were used to investigate between-meal postprandial variations (Matthews et al. 1990). Two-way ANOVA, using subject and meal as the independent variables, was used to investigate significant differences of these summary variables. Post-hoc statistical analysis was completed using the least significance difference, to determine significant differences between group means (Snedecor \& Cochran, 1989).

\section{Results}

Postprandial plasma triacylglycerol, TRL-triacylglycerol and plasma apoB-48 concentrations following the three test meals are presented in Fig. 1. Repeated-measures ANOVA showed that plasma triacylglycerol, TRL-triacylglycerol and apoB-48 concentrations increased significantly ( $P=0.0001$ ) following all three test meals. However there was no significant difference in the postprandial responses between the three test meals. A bi-phasic postprandial response was observed following all meals. ApoB-48 concentrations in the TRL and TPL were not significantly different between meals, therefore the results are not shown. The AUC, maximum postprandial plasma concentrations and time to maximum postprandial plasma triacylglycerol concentration of postprandial plasma triacylglycerol, TRL-triacylglycerol and apoB-48 concentrations were not significantly different between meals.

The changes in plasma NEFA concentrations during the postprandial response following the three test meals are presented in Fig. 2(a). Plasma NEFA concentrations were significantly $(P=0.0001)$ reduced following meal ingestion and returned to near fasting levels $6 \mathrm{~h}$ postprandially. There was a significant $(P=0.019)$ difference in the postprandial plasma NEFA response between meals, plasma NEFA concentrations were reduced to a lesser extent following the high-MUFA meal. Fasting NEFA concentrations and the IAUC of postprandial plasma NEFA concentrations are presented in Fig. 2(b). Fasting plasma NEFA concentrations were different, but not significantly, before the test meals, while the postprandial NEFA IAUC was significantly 

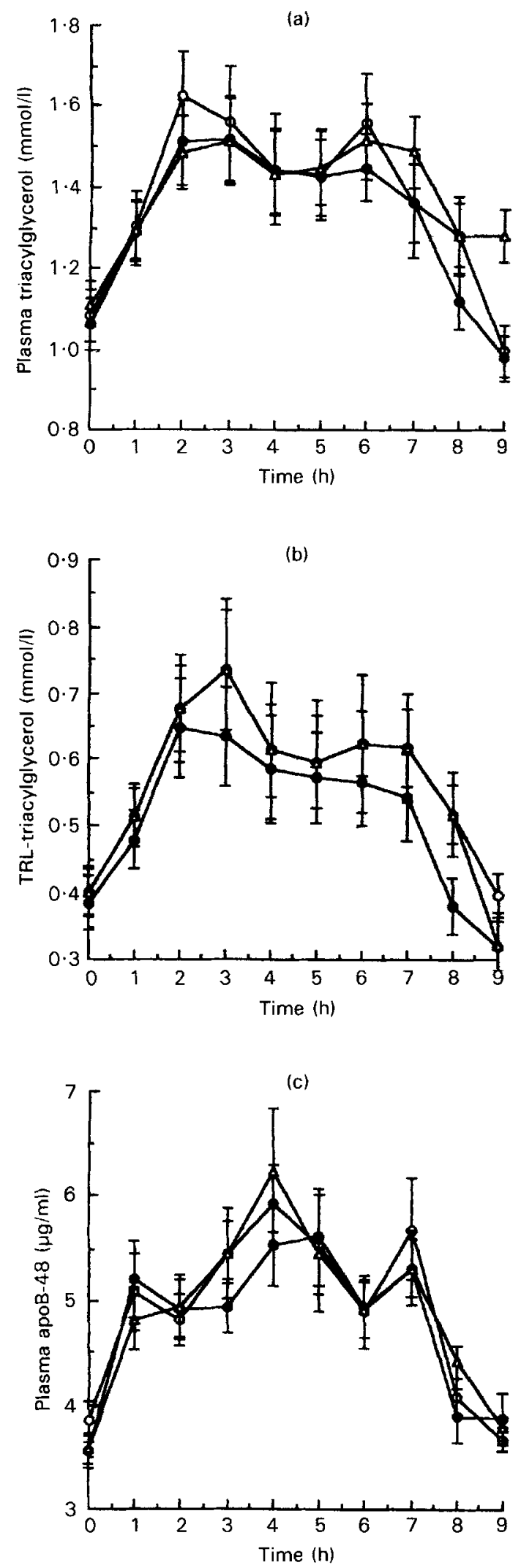

Fig. 1. Postprandial concentrations of (a) plasma triacylglycerol, (b) triacylglycerol-rich lipoprotein (TRL)-triacylglycerol and (c) plasma apolipoprotein (apo) B-48 following test meals containing high $(\mathrm{O})$, medium $(\bullet)$ and low $(\triangle)$ levels of monounsaturated fatty acids. Vaiues are means for thirty subjects, with their standard errors represented by vertical bars.
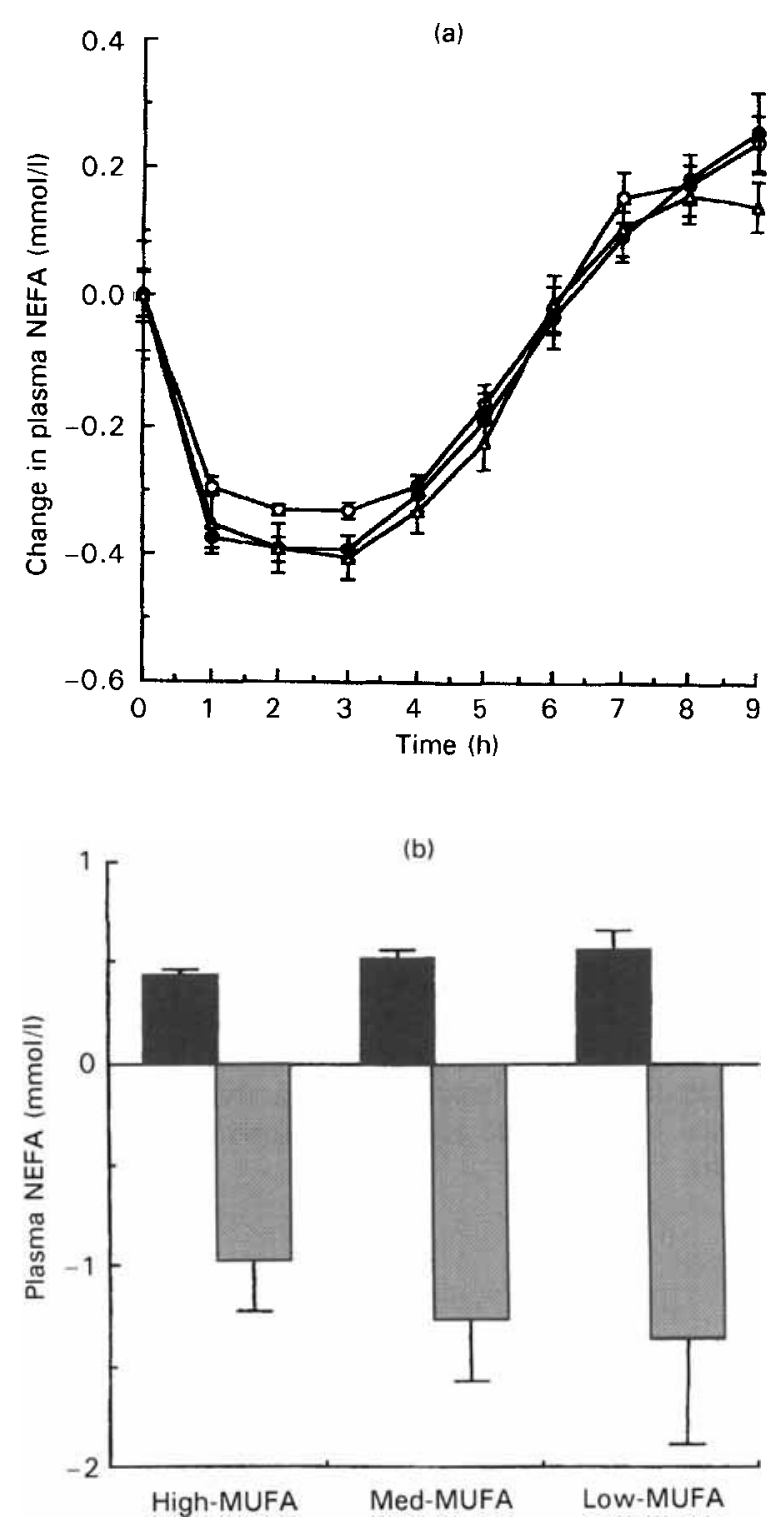

Fig. 2. (a) Change in postprandial plasma non-esterified fatty acid (NEFA) concentrations and (b) fasting NEFA concentrations ( $\square$ ) and incremental areas under the postprandial NEFA curves following test meals containing high $(O)$, medium $(O)$ and low $(\triangle)$ levels of monounsaturated fatty acids (MUFA). Values are means for thity subjects, with their standard errors represented by vertical bars.

$(P=0.0332)$ lower following the high-MUFA meal. Stepwise multiple regression analysis showed that fasting plasma NEFA concentration $(\beta-5.81 ; P=0.0001)$, followed by fasting plasma triacylglycerol concentrations $(\beta \quad 0.735 ; P=$ $0.078)$, hepatic lipase activity $(\beta-0.006 ; P=0.025)$ and meal MUFA content $(\beta-0.182 ; P=0.078)$ were the factors which determined the postprandial NEFA IAUC.

Postprandial plasma glucose, insulin, total plasma cholesterol and HDL-cholesterol concentrations were not significantly different between meals, therefore these results are not presented. Mean postprandial post-heparin lipoprotein lipase activities were not significantly different following the high-, medium- and low-MUFA test meals (334 (SD 13), 342 (SD 13), 327 (SD 12) $\mathrm{mU} / \mathrm{ml}$ respectively). 


\section{Discussion}

The postprandial lipaemic response is known to affect the pathogenesis and progression of CHD (Groot et al. 1991; Karpe et al. 1994). The magnitude of this response is affected by physiological factors including fasting lipid concentrations, age, sex, body weight and lipoprotein lipase activity (Roche \& Gibney, 1995). Dietary factors, particularly habitual dietary fat composition and the composition of fat in the test meal have been shown to affect the magnitude of the postprandial triacylglycerol response (Weintraub et al. 1988; De Bruin et al. 1993; Zampelas et al. 1994), but these studies did not measure the postprandial NEFA response. The present study investigated whether changing the proportions of MUFA and SFA in acute test meals affected postprandial triacylglycerol and NEFA metabolism. The test meals used in the present study were designed to reflect habitual dietary fatty acid composition in northern and southern Europe (Gregory et al. 1990; Kafatos \& Mamalakis, 1993), thereby investigating the biochemical basis of the beneficial effect associated with the southern European diet, which is rich in MUFA. The present study clearly demonstrated that the replacement of SFA with MUFA in acute test meals does not influence postprandial plasma triacylglycerol, TRL-triacylglycerol, apoB-48, insulin, glucose and cholesterol concentrations or post-heparin lipoprotein lipase activity. The results of this experiment are important and noteworthy because the effect of acute test meals which provide different proportions of MUFA and SFA on postprandial lipid metabolism has never been investigated. Furthermore there were two aspects of the study which merit discussion: first, a consistent bi-phasic triacylglycerol response was shown; second, the mean MUFA content may have affected postprandial NEFA metabolism.

In the present study all subjects showed a consistent biphasic postprandial triacylglycerolaemic response, irrespective of test meal fatty acid composition. Studies investigating the effect of ingesting a single test meal following an overnight fast usually show a non-phasic triacylglycerol response (Roche \& Gibney, 1996), whilst meals consumed midday or in the early evening cause a biphasic triacylglycerol response, the first peak being attributed to the previous meal and the second peak representing the triacylglycerol of the test meal (Zampelas 1994; Fielding et al. 1996). In the present study subjects had fasted overnight for at least $12 \mathrm{~h}$, therefore it is unlikely that the early triacylglycerol peak could have been derived from their previous meal. The second triacylglycerol peak could have been due to hepatically-derived VLDL secretion, which occurs $4-5 \mathrm{~h}$ following meal ingestion (Schneeman $e t$ al. 1993). The test meals contained fructose, which is known to increase the postprandial triacylglycerol response (Jeppsen et al. 1995) by stimulating hepatic VLDL production (Cohen $\&$ Schall, 1988). Therefore the fructose component of the test meal could have contributed to the second triacylglycerol peak. A recent study in this laboratory investigated this hypothesis by comparing the effect of a test meal identical to that used in the present study with one that provided the same amount of fat but a lower level of carbohydrate $(136 v .17 \mathrm{~g})$ (Shishehbor, 1997). The high-carbohydrate meal (like that used in the present study) evoked a mean bi-phasic response, while the low-carbohydrate meal evoked a mono-phasic triacylglycerol response. Therefore the carbohydrate component of the test meal represents the most likely explanation for the consistent bi-phasic response seen in this study.

In the present study the magnitude of the postprandial NEFA response differed significantly following the test meals. There was less suppression of plasma NEFA levels following the high-MUFA meal but this difference cannot be attributed to the fatty acid composition of the test meal because there was a non-significant difference in fasting plasma NEFA concentrations. Regression analysis showed that fasting NEFA levels were the most important determinant of the postprandial NEFA IAUC, while the level of MUFA in the test meal had least effect on NEFA IAUC. There are three possible mechanisms to explain the lesser suppression of postprandial NEFA concentrations in the early postprandial phase following the high MUFA test meal: (1) lesser inhibition of hormone-sensitive lipase; (2) greater 'over-spill' of fatty acids following TRL-triacyglycerol hydrolysis; or (3) reduced adipose tissue NEFA uptake. The actual reduction in NEFA concentrations $1 \mathrm{~h}$ following all meals, and insulin concentrations, were not different between meals. Therefore it is unlikely that there were any differences in hormone-sensitive lipase inhibition between meals. Since fasting NEFA concentrations were higher before the high-MUFA test meal and the actual reduction in NEFA concentrations was equal following all meals, the lesser suppression (or greater postprandial levels) of plasma NEFA concentrations following the high-MUFA meal was probably due to the greater initial concentration. Otherwise, the difference between meals could have been due to faster postprandial processing of TRL, with subsequent greater NEFA release into the circulation. Coifferier et al. (1987) showed that unsaturated triacylglycerols are hydrolysed more rapidly than those containing SFA. Therefore it may be that triacylglycerols containing oleic acid are hydrolysed more efficiently than saturated triacylglycerols, with greater over-spill of NEFA into the circulation. Therefore any difference which might be due to the fat composition of the test meal would relate to a greater rate of chylomicron hydrolysis in the early postprandial phase which may explain the higher NEFA concentrations for the first $3 \mathrm{~h}$ following the high-MUFA test meal. There has been no investigation of the effect of different fats on adipose tissue NEFA uptake, therefore it would be purely speculative to attribute the difference to this possible mechanism.

In conclusion, this study shows that the replacement of SFA with MUFA in acute test meals does not affect postprandial TRL metabolism. There may have been a small effect on postprandial NEFA metabolism, but the extent of this could not be clearly defined due to the nonsignificant difference in fasting plasma NEFA concentration before the test meals.

\section{Acknowledgement}

This project was funded by the European Union, Contract Number AIR2-CT93-1430. 


\section{References}

Binnert C, Pachiaudi C, Beylot M, Croset M, Cohen R, Riou JP \& Laville M (1996) Metabolic fate of an oral long-chain triglyceride load in humans. American Journal of Physiology 270, E445-E450.

Byrne CD, Brindle NPJ, Wang TWM \& Hales CN (1991) Interaction of non-esterified fatty acid and insulin in control of triacylglycerol secretion by Hep G2 cells. Biochemical Joumal 280, 99-104.

Cohen JC \& Schall R (1988) Reassessing the effects of simple carbohydrates on the serum triglyceride responses to fat meals. American Journal of Clinical Nutrition 48, 1031-1034.

Coifferier E, Paris R \& Lecerf J (1987) Effects of dietary saturated and polyunsaturated fat on lipoprotein lipase and hepatic triglyceride lipase activity. Comparative Biochemistry and Physiology 88B, 187-192.

Coppack SW, Jensen MD \& Miles JM (1994) In vivo regulation of lipolysis in humans. Journal of Lipid Research 35, 177-193.

De Bruin TWA, Brouwer CB, van Linde-Sibenius M, Jansen H \& Erkelens DW (1993) Different postprandial metabolism of olive oil and soybean oil: a possible mechanism of the high-density lipoprotein conserving effect of olive oil. American Journal of Clinical Nutrition 58, 477-483.

Fielding BA, Callow J, Owen RM, Samara JS, Matthews DR \& Frayn KN (1996) Postprandial lipaemia: the origin of an early peak studied by specific fatty acid intake during sequential meals. American Journal of Clinical Nutrition 63, 36-41.

Frayn KN, Shadid S, Hamlani R, Humphreys SM, Clark ML, Fielding BA, Boland O \& Coppack SW (1994) Regulation of fatty acid movement in human adipose tissue in the postabsorptive-to-postprandial transition. American Journal of Physiology 266, E308-E317.

Frayn KN, Wiliams CM \& Amer P (1996) Are increased plasma non-esterified fatty acid concentrations a risk marker for coronary heart disease and other chronic diseases? Clinical Science 90, 243-253.

Gregory J, Foster K, Tyler H \& Wiseman M (1990) The Dietary and Nutritional Survey of British Adults. London: H.M. Stationery Office.

Groot PHE, van Stiphout WAHJ, Krauss XH, Jansen H, van Tol A, van Ramnhorst E, Chin-On S, Hofman A, Cresswell SR \& Havekes L (1991) Postprandial lipoprotein metabolism in normolipidemic men with and without coronary heart disease. Arteriosclerosis and Thrombosis 11, 653-662.

Grundy SM \& Mok HYI (1976) Chylomicron clearance in normal and hyperlipidemic men. Metabolism 25, 1225-1239.

Jeppsen J, Chen Y-DI, Zhou M-Y, Wang T \& Reaven GM (1995) Effect of variations in oral fat and carbohydrate: effects of fructose. American Journal of Clinical Nutrition 62, 787-791.

Kafatos A \& Mamalakis G (1993) Changing patterns of fat intake in Crete. European Journal of Clinical Nutrition 47, S21-S24.

Karpe F, Steiner G, Uffelman K, Olivecrona T \& Hamsten A (1994) Postprandial lipoproteins and progression of coronary atherosclerosis. Atherosclerosis 106, 83-97.

Keys A, Menotti A, Karvonen MJ, Aravanis C, Blackburn H, Buzina R, Djordjevic BS, Doutas AS, Fidanza I, Keys MH, Kromhout D, Nedeljkovic S, Punsar S, Seccareccia F \& Toshima $\mathrm{H}(1986)$ The diet and 15-year death rate in the seven countries study. American Journal of Epidemiology 124, 903-915.
Lovegrove JA, Isherwood SG, Jackson KG, Williams CM \& Gould BJ (1996) Quantitation of apolipoprotein B-48 in triacylglycerol-rich lipoproteins by a specific enzyme-linked immunoabsorbent assay. Biochimica et Biophysica Acta 1301, 221-229.

Matthews JNS, Altman DG, Campbell MJ \& Royson P (1990) Analysis of serial measurements in medical research. British Medical Journal 300, 230-235.

Mensink RP (1992) Dietary monounsaturated fatty acids and serum lipoprotein levels in healthy subjects. Atherosclerosis 110, 65-68.

Mensink RP \& Katan MB (1989) Effect of a diet enriched with monounsaturated or polyunsaturated fatty acids on levels of low-density lipoprotein cholesterol in healthy women and men. New England Journal of Medicine 321, 436-441.

Nilsson-Ehle P \& Schotz MC (1976) A stable radioactive substrate emulsion for assay of lipoprotein lipase. Journal of Lipid Research 17, 536-541.

Patsch JR, Miesenbock G, Hopferwieser T, Muhlberger V, Knapp E, Dunn JK, Gotto AM \& Patsch W (1993) Relation of triglyceride metabolism and coronary artery disease. Arteriosclerosis and Thrombosis 12, 13361345.

Peel AS, Zampelas A, Williams CM \& Gould BJ (1993) A novel antiserum specific to apolipoprotein B-48: application in the investigation of postprandial lipidaemia in humans. Clinical Science 53, 521-524.

Roche HM \& Gibney MJ (1995) Postprandial triacylglycerolaemia-nutritional implications. Progress in Lipid Research 34, 249-266.

Roche HM \& Gibney MJ (1996) Postprandial triacylglycerolaemia: the effect of low-fat dietary treatment with and without fish oil supplementation. European Journal of Clinical Nutrition 50, 249-266.

Schneeman BO, Kotite L, Todd KM \& Havel RJ (1993) Relationships between the responses of triglyceride-rich lipoproteins in blood plasma containing apolipoproteins B-48 and B-100 to a fat-containing meal in normolipidemic humans. Proceedings of the National Academy of Sciences USA 90, 2069-2073.

Shishehbor F (1997) Nutritional factors affecting postprandial lipaemia. PhD Thesis, University of Dublin.

Snedecor GW \& Cochran WG (1989) Analysis of variance. In Statistical Methods, 8th ed., pp. 217-246. Ames, IA: Iowa State University Press.

Stampfer MJ, Krauss RM, Ma J, Blanche PJ, Holl LG, Sacks FM \& Hennekens CH (1996) A prospective study of triglyceride level, low-density liporotein particle diameter, and risk of myocardial infarction. Journal of the American Medical Association 276, 882-888.

Weintraub MS, Zechner R, Brown A, Eisenberg S \& Breslow JL (1988) Dietary polyunsaturated fats of the $\omega-6$ and $\omega-3$ series reduce postprandial lipoprotein levels. Journal of Clinical Investigation 82, 1884-1893.

Zampelas A, Peel AS, Gould BJ, Wright J \& Williams CM (1994) Polyunsaturated fatty acids of the $n-6$ and $n-3$ series: effects on postprandial lipid and apolipoprotein levels in healthy men. European Journal of Clinical Nutrition 48, 842848. 